\title{
Neue ISO 9001 Revision - und ihre Bedeutung für die Messtechnik in der Produktion
}

\author{
Dietrich Imkamp ${ }^{1}$, Thomas Kistner ${ }^{1}$ \\ ${ }^{1}$ Carl Zeiss Industrielle Messtechnik GmbH \\ ZEISS Gruppe, 73446 Oberkochen, Germany
}

\begin{abstract}
Zusammenfassung
Ende 2015 wurde die neue Revision der DIN EN ISO 9001 für Qualitätsmanagementsysteme veröffentlicht. In diesem weit verbreiteten Standard finden sich verschiedene Anforderungen an messtechnische Systeme, die zur Prüfung von Produkten in der industriellen Produktion verwendet werden. Anhand der Entwicklung dieses Standard werden die Anforderungen an die Messtechnik und die Änderungen der neue Revision vorgestellt.
\end{abstract}

Keywords: Fertigungsmesstechnik, Qualitätsmanagement, Prüfmittel, Rückführung, Messunsicherheit

\section{Einführung: Prüfen durch Messen}

In der modernen industriellen Produktion spielen genormte Qualitätsmanagementsysteme eine wichtige Rolle. Die bekannteste Norm ist die DIN EN ISO 9001 [1], da es sich bei dieser Norm um den am weitesten verbreitet Standard für Qualitätsmanagementsysteme handelt [2]. Diese Norm fordert eine Produktion unter so genannten „beherrschten Bedingungen". Damit ist unter anderem die Durchführung von Überwachungs- und Messtätigkeiten gemeint, um zu verifizieren, dass die Kriterien zur Steuerung von Prozessen oder Ergebnissen sowie die Annahmekriterien für Produkte erfüllt wurden.

In der Begriffsnorm DIN EN ISO 9000 [3] zu Qualitätsmanagementsystemen wird das Verifizierung als Bestätigung durch Bereitstellung eines objektiven Nachweises, dass festgelegte Anforderungen erfüllt worden sind, bezeichnet. Dieser objektive Nachweis kann unter anderem durch eine Messung erbracht werden. Die Bestimmung der Erfüllung von festgelegten Anforderungen wird als Prüfen bezeichnet. Damit wird der Bogen zu der gebräuchlicheren Begriffsweise des Prüfen durch Messen geschlagen.

Dabei darf man aber nicht vergessen, dass es in der Produktion sowohl Prüfungen gibt, die nicht auf Messungen basieren, als auch Messungen, die nicht zur Prüfung dienen (Abbildung 1). Dieser Beitrag beschränkt sich auf die Messungen für Prüfaufgaben.

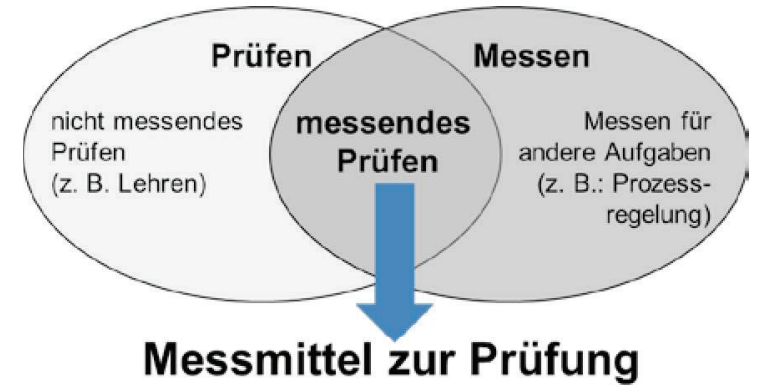

Abb.. 1: Prüfen und Messen in der Produktion.

\section{Messunsicherheit und Toleranzverständnis}

Bei der Verwendung von Messergebnissen als objektiver Nachweis, dass festgelegte Anforderungen erfüllt worden sind, ist zu berücksichtigen, dass jedes Messergebnis eine Messunsicherheit aufweist. Diese Messunsicherheit lässt sich nicht vermeiden, da der Messprozess wie der Produktionsprozess Störeinflüssen ausgesetzt ist, so dass das vom Messsystem angezeigte Messergebnis vom tatsächlichen Wert der Messgröße abweicht. Zusätzlich kann die Unsicherheit durch Unzulänglichkeiten bei der Spezifikation der Messgröße vergrößert werden (z. B. Filterparameter bei einer Formmessung). Das wird als Spezifikationsunsicherheit bezeichnet.

Diese Messunsicherheit muss ermittelt [4] werden und bei der Prüfung der Toleranz der Messgröße berücksichtigt werden. 
Es ist naheliegend, dass die Messunsicherheit wesentlich kleiner als die Toleranz sein muss, damit die Messgröße beispielsweise als Kennwert eines Prozesses das tatsächliche Prozessgeschehen wiederspiegelt. Gemäß der "goldenen Regel“ der Messtechnik soll die Messunsicherheit ein Zehntel, im äußersten Fall ein Fünftel der Toleranz nicht überschreiten [5], [6]. In diesem Fall wird die Messunsicherheit als vernachlässigbar angesehen. Der Annahmebereich entspricht in diesem Fall dem Toleranzbereich. Bei diesem Toleranzverständnis wird eine Überschreitung der Toleranzgrenzen um die Messunsicherheit hingenommen. Diese Überschreitung ist nur zu vermeiden, wenn die Toleranz um die Messunsicherheit verringert wird. Bei diesem als „Entscheidungsregeln" bezeichneten Toleranzverständnis wird der Annahmebereich um die Messunsicherheit an den Toleranzgrenzen verkleinert. Dieses Verständnis ist genormt [7] und gilt immer, sofern nichts anderes vereinbart wurde (Abbildung 2).

\section{"Goldene Regel":}

Eine (kleine) Überschreitung der Toleranz um die Messunsicherheit wird zugelassen.

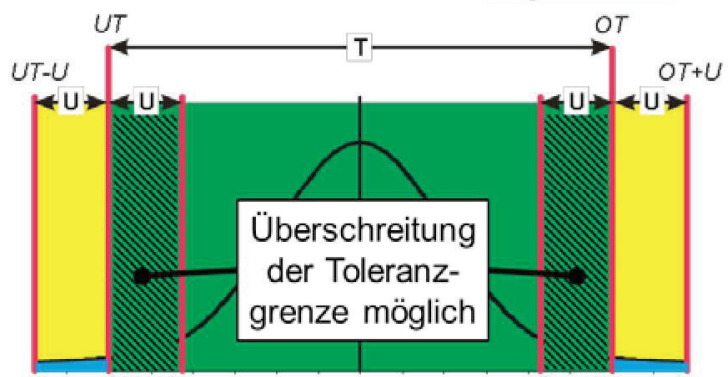

"Entscheidungsregeln ISO 14253":

Eine Überschreitung der Toleranz um die

Messunsicherheit wird nicht zugelassen.

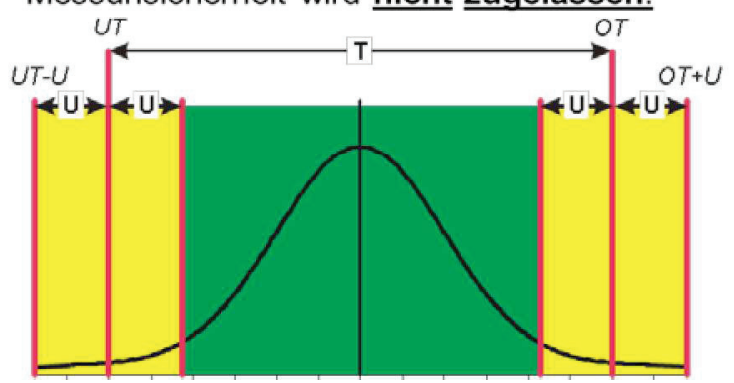

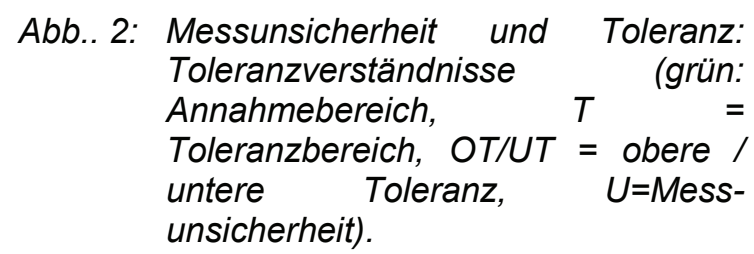

Die Messunsicherheit von Messergebnissen ist sowohl bei der Auswahl einer geeigneten Messtechnik im Rahmen der Prüfplanung als auch bei ihrer Qualifizierung und fortdauernden Überwachung zu berücksichtigen.

\section{Anforderungen der Norm (2015)}

Aufgrund der großen Bedeutung der Messtechnik bei der Prüfung industriell hergestellter Produkte und der dabei erforderlichen Berücksichtigung der Messunsicherheit, gibt es viele Anforderungen an die Messtechnik in der Norm DIN EN ISO 9001 für Qualitätsmanagementsysteme.

Seit ihrer Entstehung lassen sich die Anforderungen dieser Norm in Anforderungen an die Anwendung von Messtechnik und in Anforderungen an die dazu verwendeten Mittel unterteilen. In der aktuellen Ausgabe sind die Anforderungen an die Anwendung im Abschnitt 8 zum Betrieb und im Abschnitt 9 zur Bewertung der Leistung $\mathrm{zu}$ finden. Im $\begin{array}{lllll}\text { Abschnitt } 7 & \text { Unterstützung werden die }\end{array}$ Anforderungen an die Messmittel definiert (Abbildung 3).

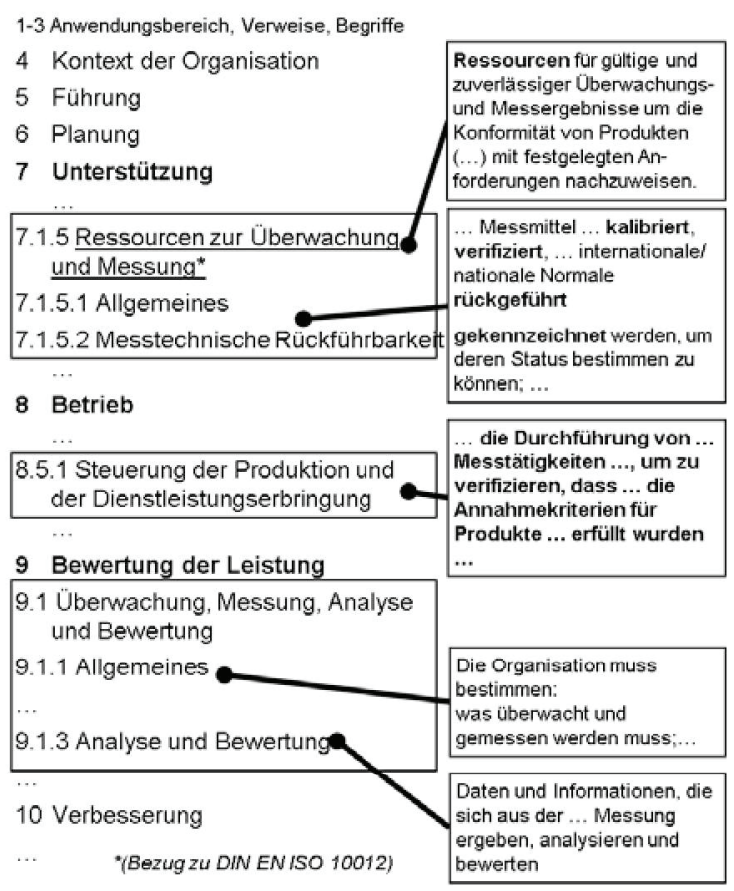

Abb.. 3: Anforderungen der DIN EN ISO 9001 bezüglich Messtechnik.

\section{Anforderungen im Vergleich}

Während die ersten Ausgaben aus den 80er und 90er Jahren die Anforderungen in Form einer Liste $\mathrm{zu}$ verschiedenen Themenbereichen darstellten [8], wurde mit der Ausgabe aus dem Jahr 2000 der so genannte „prozessorientierte Ansatz" zur Strukturierung der Anforderungen eingeführt. 
Die Basis hierzu bildet der PDCA-Zyklus. Die Abkürzung PDCA steht dabei für „Plan Do Check Act-Zyklus" (deutsch: Planen - Tun Überprüfen - Umsetzen) und wird nach seinem Entwickler auch als "Deming-Kreis“ bezeichnet. Er beschreibt einen vierphasigen Problemlösungsprozess zur kontinuierlichen Verbesserung [9]. Er ist ein grundlegender Bestandteil des Qualitätsmanagementstandards DIN EN ISO 9001.

Die Anforderungen der Norm werden den verschiedenen Phasen dieses Zyklus zugeordnet. Das hat sich in der Ausgabe der DIN EN ISO 9001 von 2015 zur Vorgängerversion von 2008 nicht grundlegend geändert. Jedoch hat sich die Grundstruktur (englisch: High Level Structure) der Ausgabe von 2015 stark verändert, um eine einheitliche Struktur für Managementsystemnormen zu entwickeln [10]. Daher finden sich die verschiedenen Anforderungen jetzt in anderen Abschnitten mit anderen Kapitelnummern.

Der Do-Phase des PDCA-Zyklus wurde bisher Abschnitt 7 (Produktrealisierung) zugeordnet. In der Ausgabe von 2015 wurde diese Phase auf die Abschnitte 7 (Unterstützung) und 8 (Betrieb) aufgeteilt. In der Check-Phase wurde der Abschnitt 8 (Messung, Analyse und Verbesserung) durch den Abschnitt 9 (Bewertung der Leistung) ersetzt. Der ActPhase wurde in der vorhergehenden Ausgabe der Abschnitt 5 (Verantwortung der Leitung) zugeordnet. Jetzt ist es der Abschnitt 10 (Verbesserung). Die Planung trägt in beiden Ausgaben die Kapitelnummer 6, wobei jedoch andere Überschriften die Abschnitte kennzeichnen (Abbildung 4).

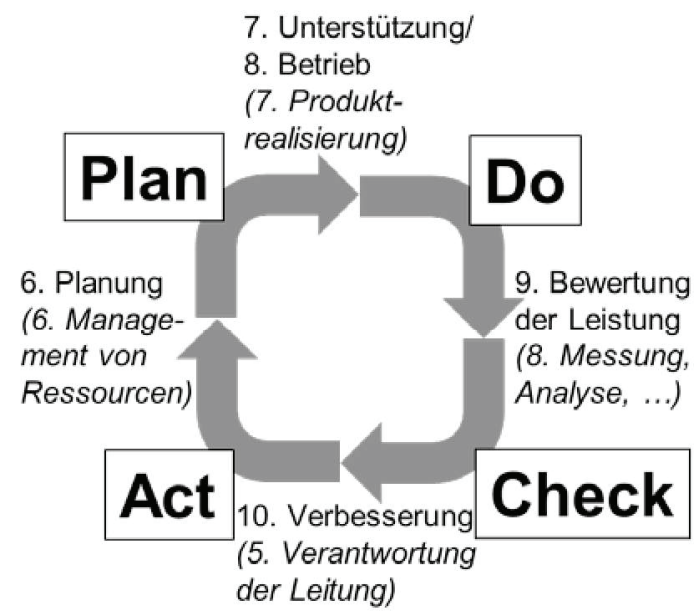

Abb.. 4: Abschnitte der ISO 9001: 2015 und 2008 (kursiv) im PDAC-Zyklus.
Die Anforderungen an die Messtechnik sind hauptsächlich in den Abschnitten zur ActPhase zu finden. In dieser Phase werden die Anforderungen an die Messtechnik in der Produktion zur Produktprüfung (Abschnitt 8.5.1) sowie die Anforderungen an die erforderlichen Ressourcen (Abschnitt 7.1.5) definiert (Abbildung 3). Dabei ist die Rückführung durch einen eigenen Abschnitt (7.1.5.2 Messtechnische Rückführung) im Vergleich zur vorherigen Ausgabe aufgewertet worden. In der Ausgabe von 2008 wurden die Anforderungen an die Ressourcen im Abschnitt 7.6 (Lenkung von Überwachungsund Messmitteln) bestimmt. Die Bezeichnung dieser Mittel wurde in der neuen Ausgabe durch „Ressourcen zur Überwachung und Messung" ersetzt. In beiden Ausgaben wird auf die DIN EN ISO 10012 [11] als Leitlinie für die Umsetzung verwiesen. In Deutschland gibt es zu diesem Thema zusätzlich die DIN 32937 [12], [13].

In beiden Ausgaben sind Anforderungen an die Messtechnik in der Check-Phase zu finden. In der Ausgabe von 2015 sind die Anforderungen hauptsächlich in Abschnitt 9.1 und in der Ausgabe von 2008 im Abschnitt 8.2.4 konzentriert. Zusätzlich finden sich Anforderungen in dieser Phase an die Analyse der durch die Messung gewonnen Daten.

In Abbildung 5 am Ende dieses Beitrags werden die hier beschriebenen Textstellen aus beiden Normenausgaben gegenübergestellt.

\section{Zusammenfassung}

Trotz der unterschiedlichen Kapitelnummerierung und Begrifflichkeit ist festzuhalten, dass sich die Anforderungen der neuen Ausgabe nicht grundsätzlich von denen der alten Ausgabe unterscheiden. Das wird besonders bei der Zuordnung der verschiedenen Kapitel zum PDCA-Zyklus deutlich (Abbildung 4). Dennoch sind die zuvor beschriebenen Veränderung natürlich zu berücksichtigen.

Durch die weitere Verallgemeinerung der Norm hat sich die neue Ausgabe noch mehr von ihrem Ursprung, der industriellen Produktion, entfernt. Diese Entwicklung vergrößert den Anwendungsbereich der Norm aber zugleich auch den Interpretationsspielraum besonders im Hinblick auf die technische Implementierung. Die verfügbaren Leitfäden [11], [12] geben hierbei jedoch weiterhin klare Vorgaben zur Umsetzung. 


\section{Abb.. 5: Vergleich der Anforderung an ein Qualitätsmanagementsystem nach DIN EN ISO 9001 von 2008 und 2015.}
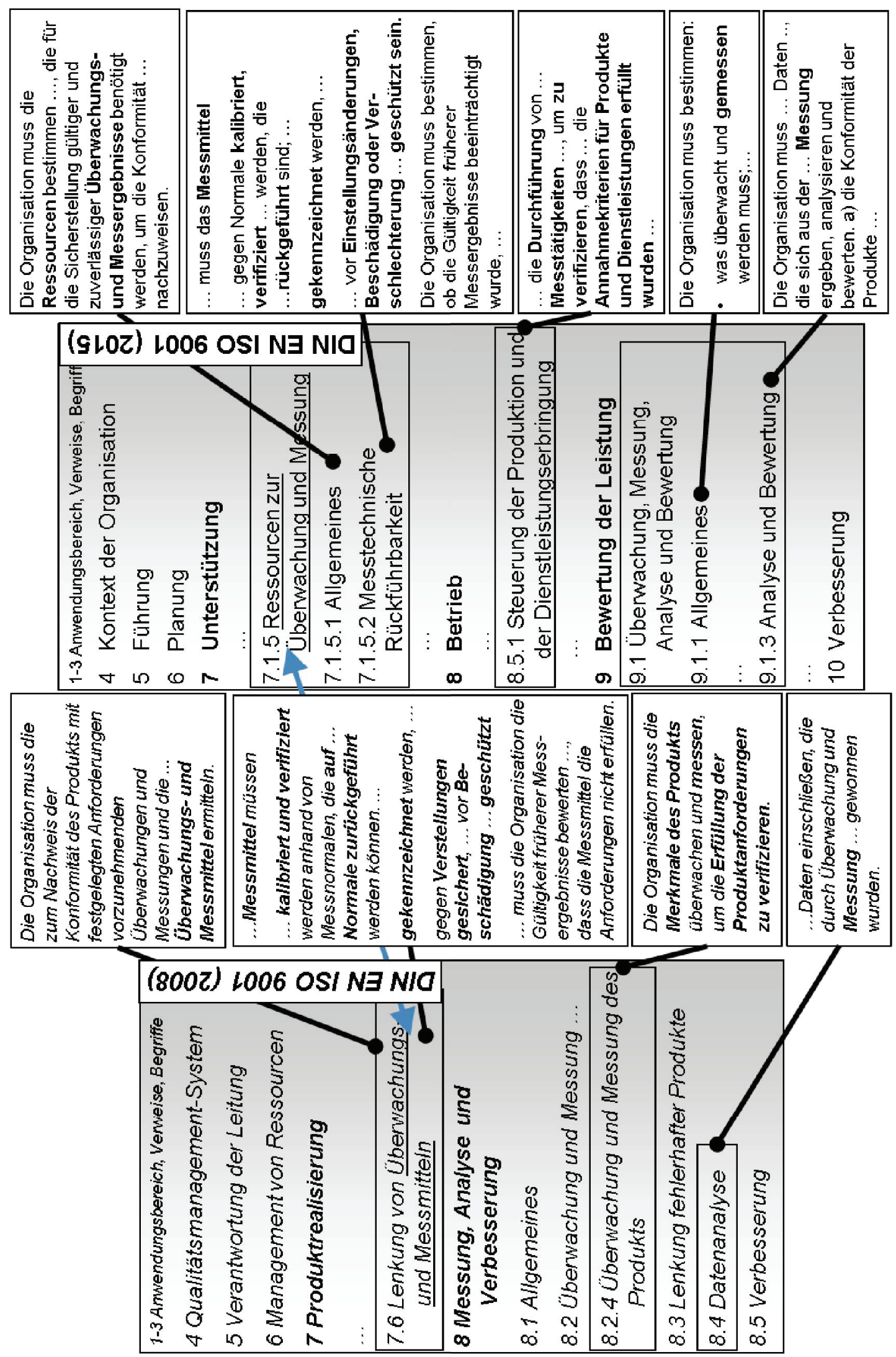


\section{Literaturnachweis}

[1] DIN EN ISO 9001: 2015

Qualitätsmanagementsysteme - Anforderungen (English: Quality management systems Requirements).

[2] ISO Survey: http://www.iso.org/iso/iso-survey (abgerufen 26.02.2016)

[3] DIN EN ISO 9000: 2015 Qualitätsmanagementsysteme - Grundlagen und Begriffe (English: Quality management systems - Fundamentals and vocabulary).

[4] Sommer, K.-H., Siebert B. R. L., :

Praxisgerechtes Bestimmen der

Messunsicherheit nach GUM, in: tm -

Technisches Messen, Oldenbourg Industrieverlag, 71 (2004) 2, S. 52-66.

[5] Dutschke, W.: Zulässige Meßunsicherheit, wt-Z. ind. Fertigung, Jahrg. 59 (1969) 12, S. 630-632.

[6] Linß, G.: Prüfplanung, in: Pfeifer, T., Schmitt, R. (Hrsg.): Masing Handbuch Qualitätsmanagement, Carl Hanser Verlag, 2007.

[7] DIN EN ISO 14253-1 (ISO 14253-1: 2013) Geometrische Produktspezifikationen (GPS) Prüfung von Werkstücken und Messgeräten durch Messen - Teil 1: Entscheidungsregeln für die Feststellung von Übereinstimmung oder Nichtübereinstimmung mit Spezifikationen (English: Geometrical product specifications (GPS) - Inspection by measurement of workpieces and measuring equipment - Part 1: Decision rules for proving conformance or nonconformance with specifications) 2013.

[8] Pfeifer, T., Imkamp, D.: Auswirkungen der DIN EN ISO 9000ff. auf die Fertigungsmeßtechnik, in: Koordinatenmeßtechnik - sicher - umfassend - zukunftsweisend, Tagung Erlangen 17.-

18.4.1996 (VDI-Berichte 1258), VDI-Verlag, Düsseldorf 1996, S.125-137, und in: Jahrbuch der VDI/VDE-GMA, VDI-Verlag, Düsseldorf 1997.

[9] Schmitt, R., Pfeifer, T.: Qualitätsmanagement Strategien, Methoden, Techniken, Carl Hanser Verlag, München 2010.

[10] Ohligschläger, J., Below, F., TÜV Rheinland Cert GmbH (Hrsg.): Die ISO 9001:2015 Interpretation der Anforderungen, ISBN (Print): 978-3-8249-1976-5, TÜV Media GmbH, Köln 2015.

[11] DIN EN ISO 10012 (ISO 10012: 2003) Messmanagementsysteme - Anforderungen an Messprozesse und Messmittel; Dreisprachige Fassung EN ISO 10012: 2003 (English: Measurement management systems Requirements for measurement processes and measuring equipment) Ausgabe: 2004-03.

[12] DIN 32937 Mess- und Prüfmittelüberwachung Planen, Verwalten und Einsetzen von Messund Prüfmitteln, 2006-07.

[13] DGQ, Deutsche Gesellschaft für Qualität (Hrsg): Prüfmittelmanagement - Planen, Überwachen, Organisieren und Verbessern von Prüfprozessen (DGQ-Band 13-61: 2003), Beuth Verlag, Berlin 2003. (Neuauflage: Kistner, A., Schäfer, B.: Prüfmittelmanagement, Carl
Hanser Verlag, München 2015, ISBN 9783446442641). 